\title{
Application of Self-Organizing Map (SOM) for Cerebral Cortex Reconstruction
}

\author{
Cheng-Hung Chuang ${ }^{1}$, Philip E. Cheng ${ }^{1}$, Michelle Liou ${ }^{1}$, Cheng-Yuan Liou ${ }^{2}$ and Yen-Ting Kuo ${ }^{2}$ \\ ${ }^{1}$ Institute of Statistical Science, Academia Sinica, \\ No. 128, Sec. 2, Academia Road, Taipei 115, Taiwan \\ chchuang@stat.sinica.edu.tw (for correspondence); pcheng@stat.sinica.edu.tw; mliou@stat.sinica.edu.tw \\ ${ }^{2}$ Department of Computer Science and Information Engineering, National Taiwan University, \\ No. 1, Sec. 4, Roosevelt Road, Taipei 106, Taiwan \\ cyliou@csie.ntu.edu.tw;r90047@ms.csie.ntu.edu.tw
}

\begin{abstract}
This paper presents the application of a self-organizing map (SOM) model for the reconstruction of cerebral cortex from MRI images. The cerebral cortex is an important tissue for many brain science or medicine related researches. Since it is difficult to extract the highly folded and buried cortical surface, we apply the SOM model to deform the easily extracted white matter surface on a layered distance map to obtain the cortical surface. The layered distance map is calculated according to the extracted white matter surface and segmented gray matter. The proposed method can reconstruct the proper cortical surface and thus make the measurement of cortical thickness easy. The simulations on T1-weighted MRI images show that the proposed algorithm is robust to reconstruct the cerebral cortex.
\end{abstract}

Keywords: self-organizing map, surface reconstruction, cerebral cortex, cortical thickness, layered distance map.

\section{Introduction}

Recently, due to the advanced magnetic resonance imaging (MRI) techniques, MRI scans are often used in the analysis of cognitive neuroscience, diseases (e.g., epilepsy, schizophrenia, Alzheimer's disease, etc.), and anatomical structures, etc. With high spatial resolution and soft-tissue contrast, MRI scans have a great potential to be used in research into anatomical structures of human brains in vivo. In general, there are three major brain tissues which can be approximately partitioned in human brains, i.e., cerebral spinal fluid (CSF), gray matter (GM), and white matter (WM). Modern anatomical MRI studies on human brains have been concentrated on the cerebral cortex, which is a thin and folded layer between GM/WM and GM/CSF interfaces $[1,2]$, i.e. the GM. Therefore, the reconstruction of cerebral cortex means to extract GM/WM and GM/CSF boundaries and rebuild its surface. There have been a lot of methods proposed in the literature to solve this problem [1-4]. These methods can be roughly classified into two categories: stochastic and morphological models.
The stochastic models [2, 3] employ labeled cortical mantle distance maps or intensity distance histograms related to the GM/WM interface so that the extraction of GM/CSF interface is needless. On the other hand, the morphological models apply the dilation of GM/WM interface [1, 4] to extract the accurate GM/CSF boundaries due to the obvious and easily extracted GM/WM interface. In the latter, a cortex is usually regarded as a double surface structure [1]. During the deformation process, the tissue partition scheme is embedded and the exterior surface following the interior surface is deformed with some constraints to find out the GM/CSF interface. Unfortunately, the extraction of accurate GM/CSF interface is difficult and still a challenge due to the image resolution and the highly folded and buried cortex joined with partial volume effects. That is, one of the major difficulties is the tissue partition problem. Simultaneously, those deformation constraints are also difficult to be adjusted to satisfy the complex situation.

There are many segmentation algorithms proposed to solve the tissue partition problem [5-7]. If the perfect or expert segmentations are prior performed, is it possible to extract the GM/CSF interface by a simple model? In our study, we focus on this issue that is how to reconstruct the cortex by a simple model on the segmented MRI images. In other words, in MRI images, there exists the sulci full of GM but no CSF can space them at intervals, i.e., the hidden cortex defined in the laminar cortex model [2]. In this paper, we apply the self-organizing map (SOM) model [8-10] which is aided with a layered distance map (LDM) to deform the GM/WM interface to find out the GM/CSF interface. In the simulation and experiment, we use a simple artificial image, a two-dimensional (2-D) T1-weighted MRI image, and three-dimensional (3-D) T1-weighted MRI data for test. Our studies on T1-weighted MRI scans show that the proposed method gains more precise results to reconstruct cortical surface. 


\section{The Problem}

In the cerebral cortex, there are many narrow and deep fissures called sulci. These concave parts sometimes contain invisible or unrecognizable CSF, which makes the reconstruction of cerebral cortex laborious. For to easily understand the problem, a T1-weighted MRI image shown in Fig. 1(a) is illustrated. This image shows a sulcus structure, i.e. the region of interest (ROI). Its segmented image is shown in Fig. 1(b), where the white color region represents $\mathrm{WM}$, the gray color region indicates GM, and the black color region is background, CSF, and other tissues. It is easy to extract the boundaries of the GM, as shown in Fig. 1(c). However, there should exist an interval within the sulcus as shown in Fig. 1(d). If the inner (WM) boundary line is deformed outward to extract the outer (GM) boundary, it will probably fail to catch the interval due to fewer extractable features inside the sulcus.

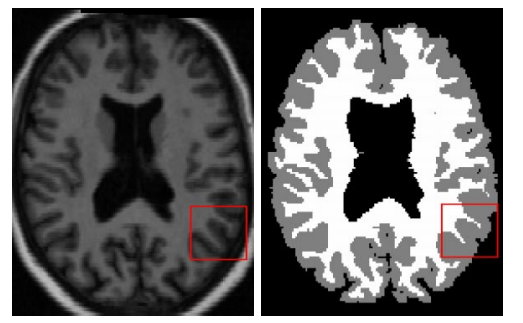

(a)

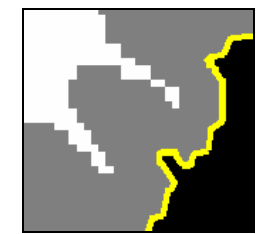

(c) (b)

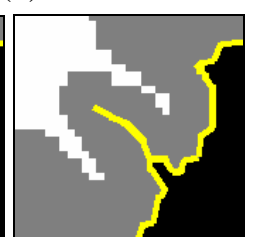

(d)
Figure 1. Illustration of a missing interval within a sulcus in a T1-weighted MRI image: (a) the raw image and the ROI, (b) its segmented image and the ROI, (c) extracted boundary of ROI, (d) ideal boundary of ROI.

\section{Methods}

\section{A. The Layered Distance Map (LDM)}

Since it is difficult to partition tissues inside sulci, the real GM surface is also hard to be extracted. Fortunately, the GM/WM boundaries are obvious and can be easily extracted. One popular way is to dilate the GM/WM interface to extract the GM/CSF boundaries. In our method, this kind of dilation strategy is also employed and assisted by a layered distance map (LDM). In [2, 3], the distance map of labeled tissues is proposed to create a secondary data structure. Accordingly, two features including intensity and distance can be obtained for statistical testing. The distance is calculated by the shortest Euclidean distance from each voxel to the GM/WM surface. However, this measure can not avoid the structure problem, e.g. crossing a CSF gap to find the shortest distance, and will cause error distance measure. Therefore, we propose the LDM to overcome this problem.

The LDMs are acquired from the segmented MRI images by a layered distance function (LDF), i.e. $L(p)$, where $p$ is an input voxel coordinate. Those voxels of GM and WM are first defined as two sets, $G$ and $W$, respectively. The LDF is formulated as

$$
L(N(q))=L(q)+1, N(q) \in G
$$

where $q$ represents labeled voxel coordinates and $N$ is a neighborhood function in which the 6-connectivity is used, i.e., the 6-neighbors of $q$ are denoted by $N(q)$. For initialization, the distance values of GM/WM interface are labeled as zeros, i.e., $L(q)=0, q \in W$. Those unlabeled GM voxels which are 6-neighbors of WM voxels are secondly labeled as ones by (1). The LDF is calculated iteratively all over the segmented MRI images until all GM voxels are labeled. Finally, the distance values of all unlabeled voxels, e.g. CSF or other tissues, are also set to zeros.

Figure 2 shows one slice of 3-D MRI data and its LDM. The various thickness in this LDM results from the 3-D MRI data structure. In Fig. 2(a), the image is partitioned into WM (white color), GM (gray color), and others (black color). The boundaries of GM are not well extracted inside the sulci. However, according to LDF, the segmented image can be processed to construct different layers in the GM as shown in Fig. 2(b). Once the LDM is constructed, it will help the GM/WM interface to deform outward to find out a proper GM/CSF interface.

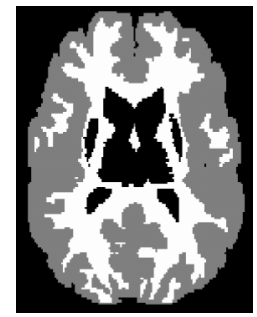

(a)

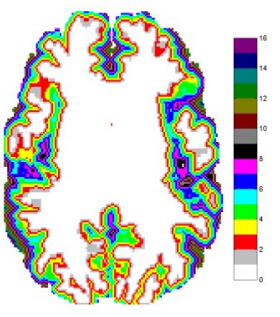

(b)
Figure 2. A segmented MRI image (a) and its layered distance map (b).

\section{B. Approximation of a Surface}

In mathematics, a surface can be defined to be a 2-D submanifold of 3-D Euclidean space. In order to approximate a surface in a simple and discrete manner, a mesh surface constructed by small triangles is defined. A triangle which is also called a face contains three edges and three vertices and encloses a plane. This portion of the plane is called the triangle interior and the plane is a 2-D surface spanned by two vectors originating at any one of the three vertices. An edge is a line segment on the boundary of a triangle. Two triangles can meet at an edge and share two vertices. Each vertex is a coordinate point in 3-D Euclidean space, i.e. $R^{3}$. The surface is smoothly approached by mesh when the triangles are very small. On the contrary, it is roughly approached when the triangles are large. 


\section{The Self-Organizing Map (SOM) Model}

The SOM model is an effective algorithm for the mapping between the neuron model and input data sets. It is a nonlinear, ordered, and smooth function that can map a high-dimensional data set onto a low-dimensional neuron model set, e.g. 3-D space data maps to a 2-D array of grid nodes. The visualization of high-dimensional data, therefore, can be easily achieved by a low-dimensional display. In our applications, it is desired to deform the GM/WM interface to find out the GM/CSF interface. The boundary voxels of WM are defined as the neuron data set. Then voxels on each layer of the LDM are established to be the input data set. The SOM model is applied to construct the mapping between the neuron data and the input data layer by layer. Finally, the $\mathrm{GM} / \mathrm{CSF}$ interface is reconstructed by the converged neuron data set.

The proposed SOM model is mathematically described in the following. It is a multi-layer SOM procedure starting with $L=1$ in the LDM, $L=1,2,3 \ldots$. First, those voxels on the layer $L-1$ whose all 6-neighbors do not belong to the layer $L$ are changed to layer $L$ in the LDM. This step is to keep those probable boundary voxels, which disconnect to next layer, all the time during SOM processing. Then, all voxels on this layer $L$ of LDM are defined as a point set $g_{L, i} \in G \subseteq R^{3}$. Each voxel represents a coordinate value $g_{L, i}$ indexed by the current processed layer $L$ and the subscript $i$. The layer starts from the lowest layer to the highest one, i.e., the layer near the GM/WM interface is processed first. The layer $L=0$ means the GM/WM interface. The GM/WM interface is also defined as a point set $w_{j} \in W \subseteq R^{3}$, where $w_{j}$ represents boundary points on GM/WM interface. In our simulation, the initial $w_{j}$ is the mesh vertices on the GM/WM interface. The best matching function is defined as

$$
f\left(w_{j}\right)=\left\|g_{L, c}-w_{j}\right\|=\min _{i}\left\|g_{L, i}-w_{j}\right\|, \quad g_{L, i} \in G, w_{j} \in W,
$$

where $w_{j}$ is the randomly selected neuron data, $g_{L, i}$ is the input set or reference data, and $g_{L, c}$ is the nearest input data point corresponding to neuron $w_{j}$. The smallest of the Euclidean distances $\left\|g_{L, i}-w_{j}\right\|$ can be made to define the best matching point. Its update function denotes

$$
w_{j^{\prime}}(k+1)=w_{j^{\prime}}(k)+\alpha(k) H(D, k)\left[g_{L, c}-w_{j^{\prime}}(k)\right],
$$

where $w_{j^{\prime}}$ including $w_{j}$ represents all neighbors around $w_{j}, k=0,1,2 \ldots$ is the iteration number, $\alpha(k) \in[0,1)$ is the learning rate, and $H$ is the neighborhood function which decreases when the distance metric $D$ and iteration $k$ increase. The update function is iteratively proceeded until the average variation of input data is less than a threshold value, $\bullet$. The Gaussian function is usually applied to be the smoothing kernel, i.e.

$$
H(D, k)=\exp \left(-\frac{D}{2 \sigma^{2}(k)}\right) \text {, }
$$

where $\sigma(k)$ is the standard deviation, i.e. the width of the smoothing kernel. The distance metric $D \equiv D\left(j^{\prime}, j\right)$ defines the distance from $w_{j^{\prime}}$ along the surface to $w_{j}$. When $j^{\prime}$ is equal to $j$, i.e. $w_{j^{\prime}}=w_{j}, D$ is zero and the neighborhood function $H$ has the maximal value 1 .

\section{Simulation and Experiment}

At first, a 2-D 32x32-pixel artificial brain sulcus image shown in Fig. 3(a) is designed, where the white color region represents WM, the gray color region indicates GM, and the black color region is CSF or other tissues. This image shows a sulcus structure full of GM and no CSF inside. The zigzag shape occurs due to low image resolution. The shortest distance is measured to evaluate the results. The extracted GM/WM and GM/CSF boundaries are shown in Fig. 3(b), where the maximal distance is 17.1 pixels, the minimal distance is 3.2 pixels, and the mean distance is 8.2 pixels. The ideal (reference) boundaries of the GM/CSF are shown in Fig. 3(c), where the maximal, minimal, and mean distances are 5.4, 3.0, and 4.1 pixels, respectively. The LDM, shown in Fig. 3(d), is first calculated according to the GM/WM boundaries, i.e. the bottom edge in Fig. 3(b). Then the $\mathrm{GM} / \mathrm{WM}$ boundary line, i.e. the initialized $w_{j}$, is deformed by the proposed SOM model with the LDM. Although there is no boundary inside the artificial brain sulcus, the deforming line is still kept inside the sulcus. The proposed method is successively proceeded 100 times to obtain an average result, as shown in Fig. 3(e), where the maximal, minimal, and mean distances are 6.2, 3.1, and 4.2 pixels, respectively. The experimental result by using the conventional SOM model (without LDM) is shown in Fig. 3(f), where no boundaries are detected inside the sulcus.

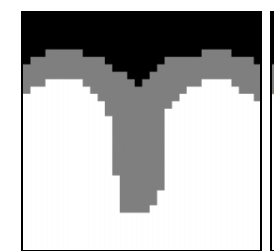

(a)

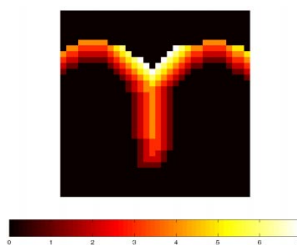

(d)

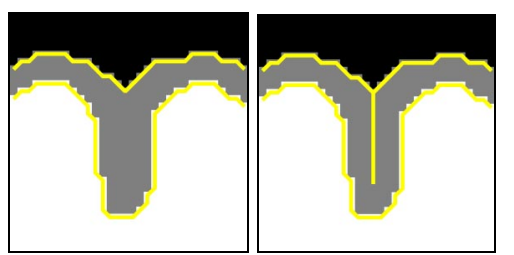

(b)

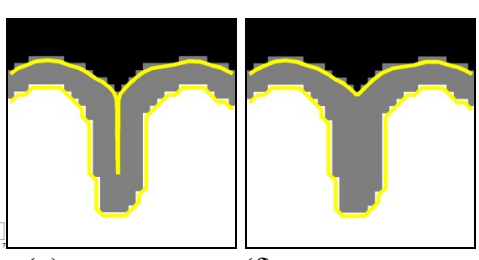

(e) (f)
Figure 3. Experiments on a 2-D artificial brain sulcus image: (a) the raw image, (b) the boundary image of (a), (c) the ideal (reference) boundary image, (d) the LDM, (e) an average result of 100 times experiments by the proposed method, (f) the result by using the conventional SOM model (without LDM). 
The second experiment is a 2-D 181x217-pixel T1-weighted MRI image with its expert segmentation result, as shown in Figs. 4(a) and 4(b). The image resolution is $1 \mathrm{~mm}$. From the expert partition result, boundary pixels of GM/WM (inner) and GM/CSF (outer) interfaces can be extracted as shown in Figs. 4(c) and 4(d), respectively, where the GM/CSF boundary contours are disconnected and some of sulci lose their boundary information. The shortest distance between GM/WM and GM/CSF interfaces is also measured. In Fig. 4(d), the maximal and mean distances are 19.9 and 3.9 $\mathrm{mm}$. The boundary contours shown in Fig. 4(c), i.e. the initialized $w_{j}$, are used to compute LDM and deform by the proposed algorithm. Fig. 4(e) shows the experimental result where the maximal and mean distances are reduced to 8.1 and $3.0 \mathrm{~mm}$. The boundary contours are clear and orderly. It is acceptable and shows the robustness of reconstruction in the sulci by the proposed method. Also, the experimental result by using SOM model without LDM is shown in Fig. 4(f), where the maximal and mean distances between GM/WM and GM/CSF interfaces are 16.6 and $3.3 \mathrm{~mm}$. It is obvious that boundary contours are disorderly by using the conventional SOM model (without LDM).

The experiment is extended to 3-D T1-weighted MRI data. To implement the proposed methods, a successful partition of MRI data into GM and WM is needed. The MRI data with expert segmentations of 43 individual structures is acquired from the Internet Brain Segmentation Repository (IBSR). The data is first resampled into a resolution of $1 \mathrm{~mm}^{3}$. One slice of this MRI data and its segmentation result are shown in Fig. 5(a) and 5(b), respectively. In Fig. 5(b), the white and gray color regions represent WM and GM, and the black color region is background, CSF, and other tissues. The problem described in Section 2 is serious in this case. The GM/WM surface shown in Fig. 5(c), is reconstructed by the isosurface extractor in the MATLAB functions and is used to be the initialized $w_{j}$ in (2) and (3). For saving the computational cost, the number of mesh vertices is reduced to about 19,000 . The GM/CSF surface reconstructed by the segmentation result is also shown in Fig. 5(d). There is almost no sulcus existing in the reconstructed GM/CSF surface. On the other hand, from the segmented MRI data, the LDM is performed by (1). The GM/CSF surface shown in Fig. $5(\mathrm{e})$ is then formed by the proposed method. It is obvious that many sulci are clearly presented. Fig. 5(f) shows the result by using the conventional SOM model (without LDM), where the reconstructed surface is smooth and only the longitudinal cerebral fissure appears.

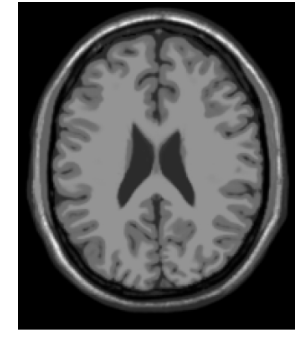

(a)

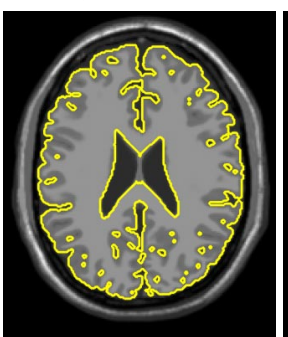

(d)

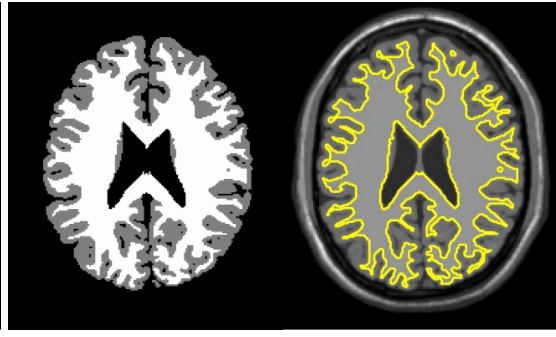

(b)

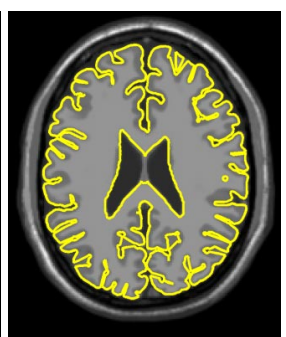

(e) (c)

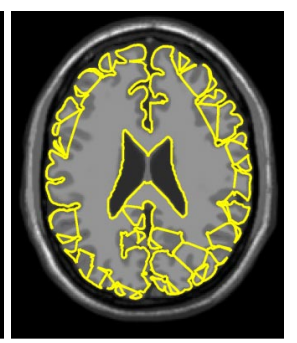

(f)
Figure 4. Experiments on a 2-D T1-weighted MRI image: (a) the raw image, (b) the expert segmentation image, (c) the inner boundary image, (d) the outer boundary image, (e) the result by the proposed method, (f) the result by using the conventional SOM model (without LDM).

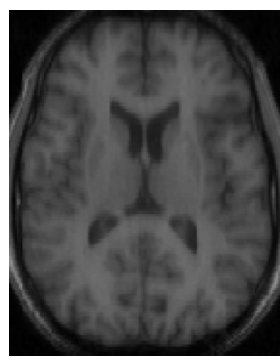

(a)

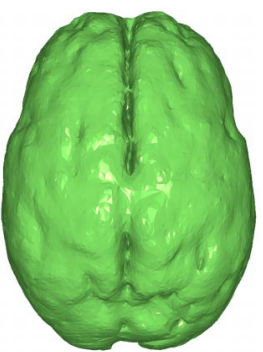

(d)

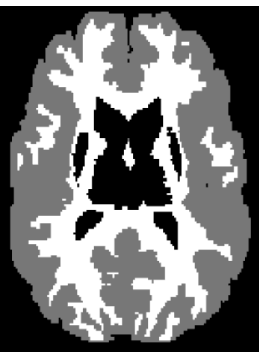

(b)

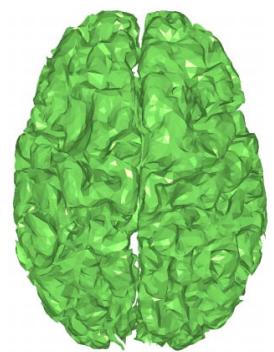

(e)

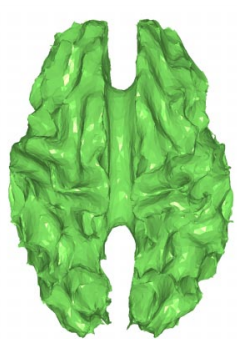

(c)

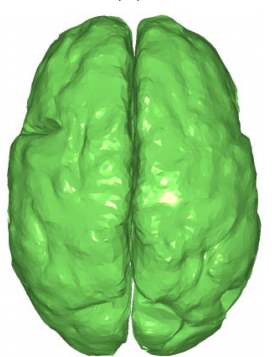

(f)
Figure 5. Experiments on 3-D T1-weighted MRI data: (a) one slice of MRI data, (b) the expert segmentation image of (a), (c) the extracted GM/WM surface from the segmentation data, (d) the extracted GM/CSF surface from the segmentation data, (e) the reconstructed GM/CSF surface by the proposed method, (f) the reconstructed GM/CSF surface by the conventional SOM model (without LDM).

\section{Conclusions}

In this paper, the SOM model is applied to reconstruct the human cerebral cortex from MRI scans. The method provides a good capability to deform the GM/WM surface outward to 
find out the GM/CSF surface. The only requirement is the segmented MRI data where GM and WM are partitioned. The layered distance map computed from the segmented data is proposed to help the SOM model to deform the GM/WM surface to a proper position of GM/CSF surface. Based on this method, even the 3-D highly folded GM/CSF surface can be reconstructed. However, in the 3-D case, one of the disadvantages is the heavy computational cost if the image resolution is very high or a large number of vertices and faces used to approximate the smooth surface are applied. Therefore, to improve the computational efficiency is the important future work. Also, from the reconstructed cerebral cortex, the measurement and analysis of the cortical thickness in association with some clinical diseases, e.g. neurodegenerative diseases and psychiatric disorders, are the advanced research topics.

\section{Acknowledgment}

The 3-D MRI data presented in this paper come from the Internet Brain Segmentation Repository (IBSR), the Center for Morphometric Analysis at Massachusetts General Hospital (http://www.cma.mgh.harvard.edu/ibsr/). This research was supported by the National Science Council, Taiwan, under the grant of NSC94-2118-M-001-012.

\section{References}

[1] D. MacDonald, et al. "Automated 3-D Extraction of Inner and Outer Surfaces of Cerebral Cortex from MRI", NeuroImage 12, pp.340-356, 2000.

[2] P. Barta, et al. "A Stochastic Model for Studying the Laminar Structure of Cortex from MRI”, IEEE Trans. Med. Imaging 24, pp. 728-742, 2005.

[3] M.I. Miller, et al. "Labeled Cortical Mantle Distance Maps of the Cingulate Quantify Differences between Dementia of the Alzheimer Type and Healthy Aging", Proc. Natl. Acad. Sci. 100(25), pp. 15172-15177, 2003.

[4] M.K. Chung, et al. "Cortical Thickness Analysis in Autism with Heat Kernel Smoothing", Neuroimage 25, pp. 1256-1265, 2005.

[5] Xiaolan Zeng, et al. "Segmentation and Measurement of the Cortex from 3-D MR Images Using Coupled-Surfaces Propagation", IEEE Trans. Med. Imaging 18, pp. 927-937, 1999.

[6] A.W. Liew, H. Yan. "An Adaptive Spatial Fuzzy Clustering Algorithm for 3-D MR Image Segmentation", IEEE Trans. Med. Imaging 22, pp. 1063-1075, 2003.

[7] $\mathrm{Z}$. Wu, et al. "Adaptive model initialization and deformation for automatic segmentation of T1-weighted brain MRI data", IEEE Trans. Biomed. Eng. 52, pp. 1128-1131, 2005.

[8] T. Kohonen. Self-Organizing Maps, 3rd ed., Springer, Berlin, 2001.
[9] C.Y. Liou, W.P. Tai. "Conformal Self-Organization for Continuity on a Feature Map", Neural Networks 12, pp. 893-905, 1999.

[10]C.Y. Liou, W.P. Tai. "Conformality in the Self-Organization Network", Artificial Intelligence 116, pp. 265-286, 2000. 